\title{
A Keratocyst in the Buccal Mucosa with the Features of Keratocystic Odontogenic Tumor
}

\author{
Kazuhiko Yamamoto*, Yumiko Matsusue, Miyako Kurihara, Yuka Takahashi and Tadaaki Kirita
}

Department of Oral and Maxillofacial Surgery, Nara Medical University

\begin{abstract}
A 74-year-old male patient consulted us for an elastic firm mass in the right buccal mucosa. CT examination revealed a well-circumscribed oval cystic lesion in the anterior region of the masseter muscle. On MRI, the lesion showed a low signal on T1-weighted image and a high signal on T2-weighted image. Aspiration biopsy demonstrated the presence of squamous cells in whitish liquid. Under the diagnosis of epidermoid cyst, the lesion was intraorally extirpated under general anesthesia. The lesion was cystic at the size of $30 \times 25 \mathrm{~mm}$. Histologically, the cyst wall was lined with parakeratinized squamous epithelium corrugated on its surface, the basal layer of which consisted of cuboidal cells showing palisading of the nuclei. Immunohistochemically, the lining epithelium was positive for CK17 and negative for CK10. The basal and suprabasal cells were labeled for Ki-67 at a relatively high rate. These features are compatible with those of keratocystic odontogenic tumor.
\end{abstract}

Keywords: Buccal mucosa, cutaneous keratocyst, epidermoid cyst, keratocystic odontogenic tumor, nevoid basal cell carcinoma syndrome, parakeratinization.

\section{INTRODUCTION}

Keratocystic odontogenic tumor (KCOT) is one of the most common odontogenic tumors of ectodermal origin. It is categorized as a benign odontogenic tumor in the WHO classification 2005 [1] because of its neoplastic potential and high recurrence rate. KCOT generally originates from the remnant of dental lamina or from basal cells of the oral epithelium [1]. It predominantly develops in the mandible or maxilla $[2,3]$, and occasionally on the gingiva as a peripheral type of manifestation [3, 4]. Interestingly, keratocysts in the buccal mucosa with similar histological features of KCOT have been reported in the recent literature [5-7]. It has not been proven whether these cysts are really odontogenic or originated from other tissues [7]; therefore, a case of keratocyst in the buccal mucosa is worthy of attention.

In this report, we present a case of keratocyst developing in the right buccal mucosa with the features of KCOT.

\section{CASE REPORT}

A 74-year-old man consulted our clinic with a complaint of swelling in the right buccal region. The patient noticed the swelling 5 years ago and had received aspiration therapy repeatedly to reduce the swelling. He was generally healthy and had no medical history. On examination, an elastic firm, movable mass of $50 \mathrm{~mm}$ was palpable in the right buccal region (Fig. 1). The overlying mucous membrane was normal.

*Address correspondence to this author at the Department of Oral and Maxillofacial Surgery, Nara Medical University, 840 Shijo-cho, Kashihara, Nara 634-8522, Japan; Tel/Fax: +81-744-29-8875;

E-mail:kazuyama@naramed-u.ac.jp
The mass was not adhesive to either the mucous membrane or the skin. The maxilla was totally edentulous and only bilateral canines and the left first premolar were present in the mandible. Hypoesthesia or palsy of the right face, trismus, swelling of cervical lymph nodes or the right parotid gland was not observed. CT examination revealed a wellcircumscribed oval cystic lesion of $35 \mathrm{~mm}$ with the density slightly less than that of muscle in the anterior region of the masseter muscle (Fig. 2a). On MRI, the lesion showed a low signal on the T1-weighted image and a heterogeneous high signal on the T2-weighted image including intermediate signal in the under portion (Fig. 2bc). Aspiration through the buccal skin revealed whitish liquid with squamous cells and shadow cells with a few neutrophils and lymphocytes in the cytological examination.

The clinical diagnosis of epidermoid cyst at the right buccal mucosa was made. The lesion was intraorally extirpated under general anesthesia through the incision along the anterior border of the mandibular ramus (Fig. 3). Detachment of the lesion from the surrounding tissue was relatively easy. Stensen's duct was not related to the lesion. The wound was closed after placing a silicon drain. Wound healing is good and no sign of recurrence has been observed for more than 4 years after the surgery.

The lesion was cystic, $30 \times 25 \mathrm{~mm}$, weighed about $6 \mathrm{~g}$ and contained brown serous liquid with a few cellular debris or keratin scale (Fig. 4). Histologically, the lesion was lined with squamous epithelium with parakeratinization corrugated on the surface (Fig. 5a). The basal layer consisted of cuboidal cells showing palisading of the nuclei and a smooth border (Fig. 5b). No daughter cysts, epithelial islands, hair 


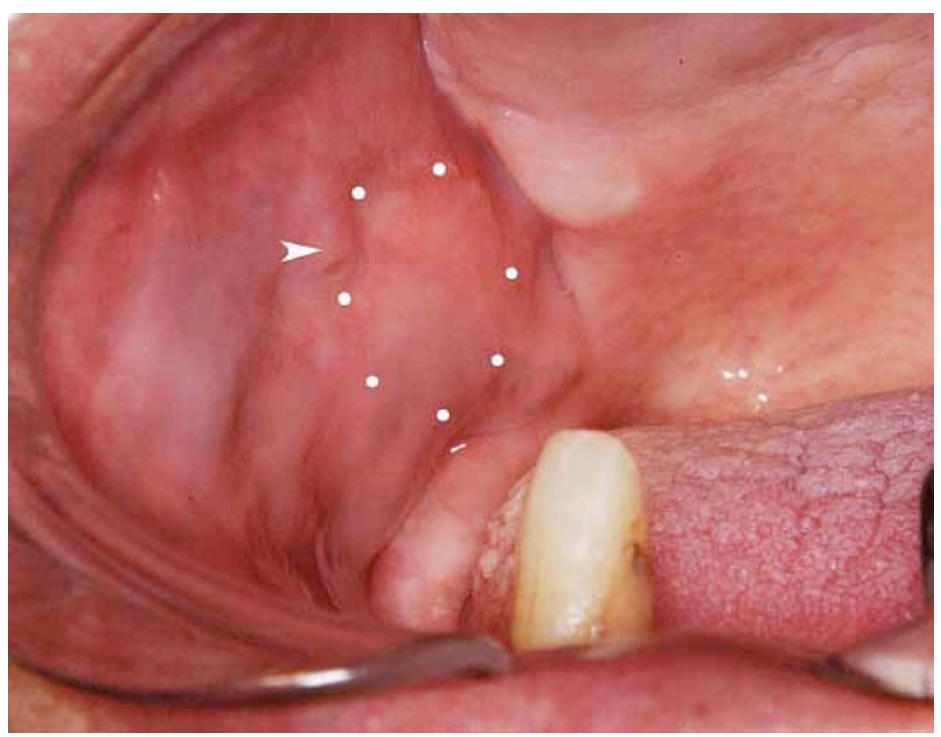

Fig. (1). Intraoral finding

Swelling is observed at the right buccal mucosa (dotted area) posterior to the orifice of Stensen's duct (arrow head).
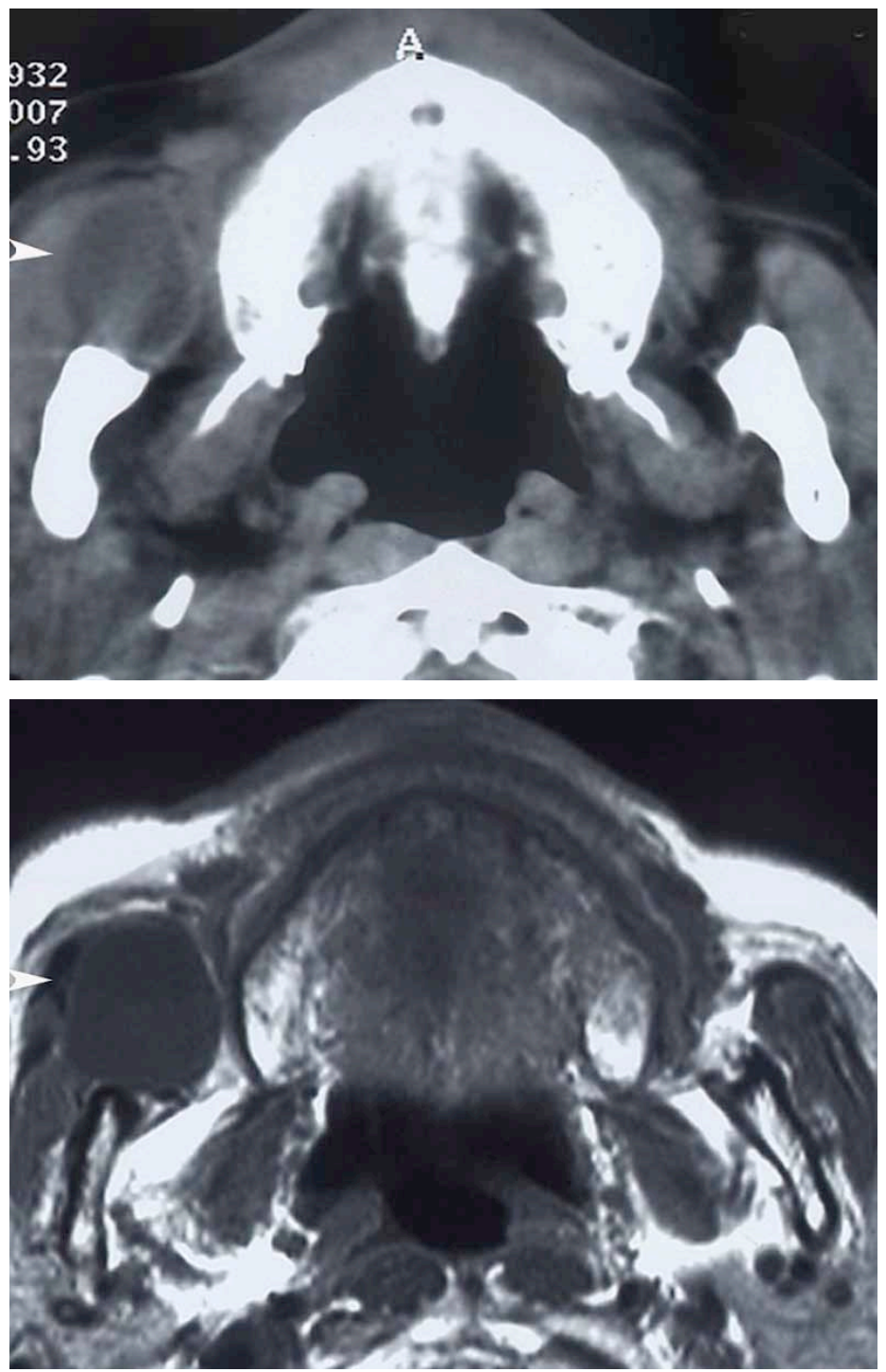

Fig. (2). Contd... 


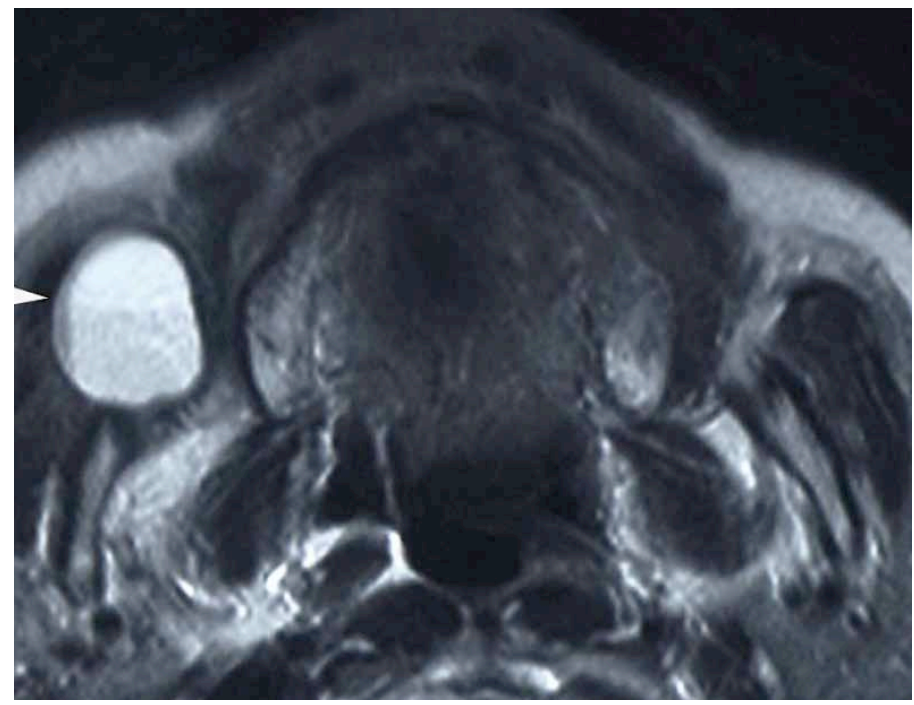

Fig. (2). Imaging

a: Axial CT, b: Axial MRI (T1WI), c: Axial MRI (T2WI)

A well-circumscribed low density mass is observed in the right buccal region anterior to the masseter muscle in CT (arrow head). It shows a low signal on T1-weighted MRI and a heterogeneous high signal on T2-weighted MRI (arrow head).

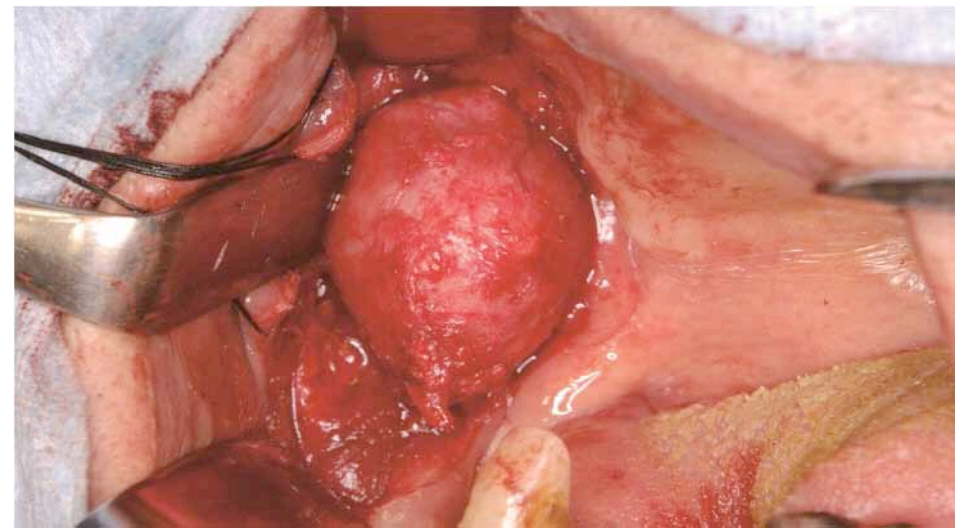

Fig. (3). Operative finding

A well-circumscribed mass is exposed anteriorly to the masseter muscle.

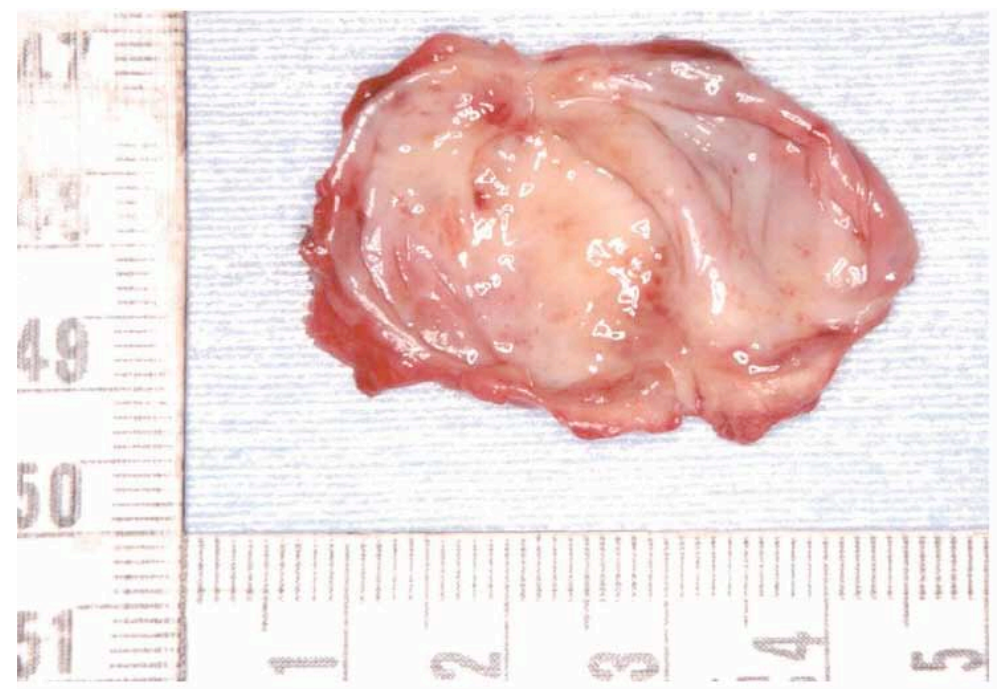

Fig. (4). Extirpated lesion

The lesion is cystic and covered with a thin smooth wall. 

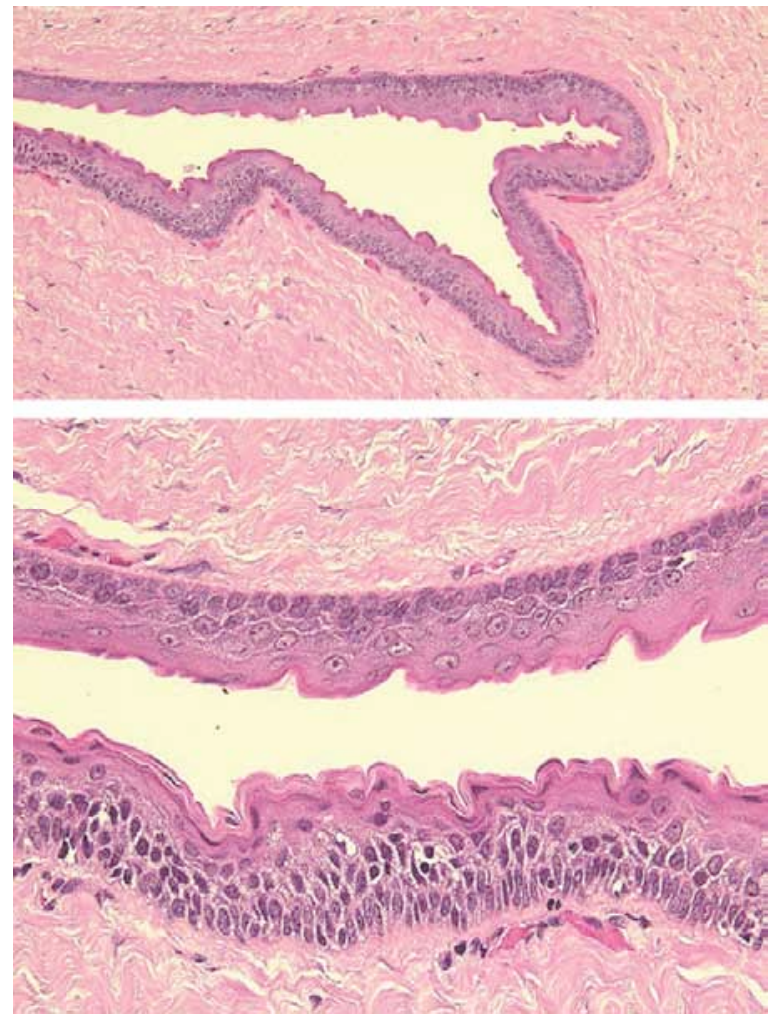

Fig. (5). Histological findings

a: The cyst wall is lined with parakeratinized squamous cells with a corrugated surface.

b: Nuclei of the cells in the basal layer are palisaded. The rete ridge of the epithelium is not evident.

follicles or sebaceous glands were found in the cyst wall. Immunohistochemically, the lining epithelium was positive for CK17 (Fig. 6a) and negative for CK10 (Fig. 6b). The basal and suprabasal cells were labeled for Ki-67 (Fig. 6c) at a relatively high rate, indicating high proliferation potential. These features are compatible with those of KCOT.

\section{DISCUSSION}

The diagnosis of KCOT is based on its characteristic histological features [8] and is not difficult for a typical keratocyst in the jawbone; however, the diagnosis of KCOT is controversial for a keratocyst in the buccal mucosa, even though it has similar histological features to KCOT. Since keratocysts in the buccal mucosa can originate from either odontogenic or epidermal tissue, a definite diagnosis cannot be made in the absence of the obvious evidence of the originating tissue. Several lesions should be differentiated from KCOT.

Epidermoid cyst with similar histological features to KCOT may develop in the buccal mucosa; however, there are several differences between KCOT and an epidermoid cyst. The preferred site of KCOT is apparently unrelated to the line of closure of the embryonic fusion plane. Histologically, the lining epithelium of KCOT shows characteristic palisading of cuboidal basal cells and parakeratinization with a corrugated surface. A different pattern of cytokeratin expression is also helpful for differential diagnosis [7]. CK10, which is specific to a cornified surface such as skin, was
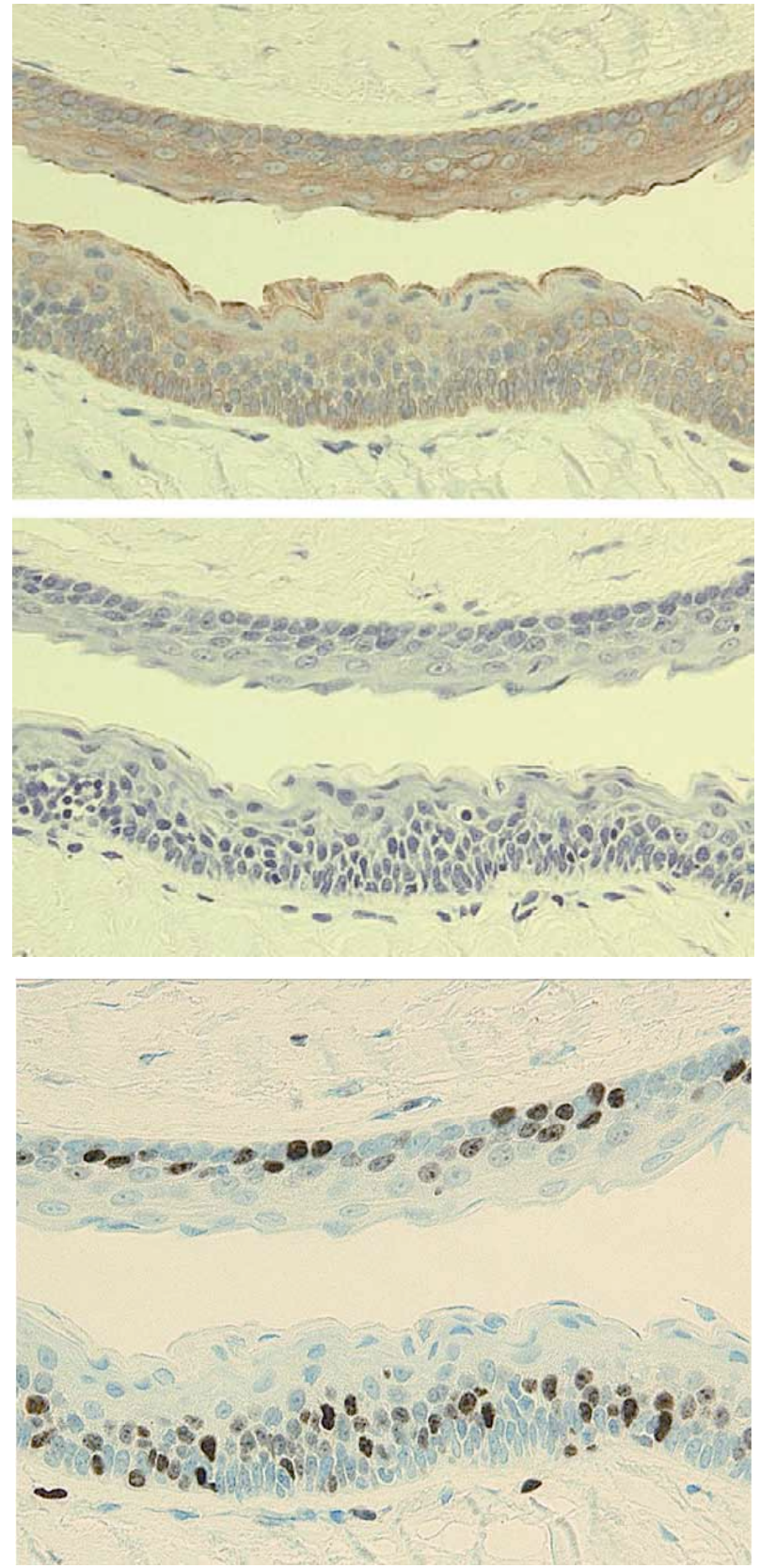

Fig. (6). Immunohistochemical findings

Immunohistochemical staining was performed using serial sections of the specimen shown in Fig. (5b).

a: CK17 (E3; Dako)

b: CK10 (DE-K10; Dako)

c: Ki-67 (MIB-1; Dako)

negative in KCOT. On the other hand, CK17 was positive in KCOT, but negative in epidermoid and orthokeratinized odontogenic cysts [9]. Ki-67-labeled cells were higher in KCOT, suggesting higher proliferation potential. Predominant suprabasal distribution of Ki-67-labeled cells is also characteristic in KCOT.

Keratocyst originating from skin adnexa such as hair follicles and sebaceous glands may ectopically occur in the buccal mucosa [7]. In addition, cutaneous keratocyst, which is generally prevalent in patients with nevoid basal cell car- 
cinoma syndrome [10,11], can also develop independently of the syndrome [12]. KCOT can be differentiated from cutaneous cystic lesions such as trichilemmal cyst and steatocystoma by its catagen pattern and the presence of pilosebaceous units, respectively [12], even if these develop in the buccal mucosa; however, cutaneous keratocysts resembling steatocystoma and lacking pilosebaceous units are quite difficult to differentiate from KCOT, since the corrugated anagen-like lining of these keratocysts is quite similar to that of KCOT [13]. Furthermore, the lining epithelium of steatocystoma, not of sebaceous cells, shows a similar pattern of cytokeratin expression to KCOT $[7,14]$. Cutaneous keratocysts rarely occur because once a true follicular cyst develops from the isthmus of a hair follicle, its lining possibly adopts a catagen pattern [13]; however, the ectopic development of a cutaneous keratocyst in the buccal mucosa cannot be completely excluded.

The mechanism by which KCOT can develop in the buccal mucosa is not well known. Since KCOT is considered to originate from the remnants of dental lamina, these cells should be displaced in the buccal mucosa and persist during embryogenesis [8]. Recent embryological reports [15,16] describing the developmental relationship between deciduous dentition and the oral vestibule may give an important clue. According to these papers $[15,16]$, the vestibular structure involved in the formation of the oral vestibule and buccal mucosa is reiteratively intermigrated with the dental epithelium around the upper molar areas during tooth formation. These findings suggest that the remnants of dental lamina entrapped in the buccal tissue during embryogenesis may develop a keratocyst with the features of KCOT. Other odontogenic lesions such as ameloblastoma and odnotoma developed in the buccal mucosa were also reported [17, 18]. This hypothesis can explain why these odontogenic lesions in the buccal mucosa uniformly develop around the parotid papilla.

In summary, a keratocyst in the buccal mucosa with the features of KCOT is reported. Although a definite diagnosis of KCOT cannot be made, an odontogenic origin is suspected from the histological features and the immunohistochemical pattern of the lesion.

\section{CONFLICT OF INTEREST}

The authors confirm that this article content has no conflicts of interest.

\section{ACKNOWLEDGEMENTS}

Declared none.

\section{REFERENCES}

[1] Philipsen HP. Keratocystic odontogenic tumour. In: Barnes L, Eveson JW, Reichart P, Sidransky D, Eds. WHO classification of tumours. Pathology and genetics of head and neck tumours. Lyon: IARC Press, 2005; pp. 306-7.

[2] Li TJ. The odontogenic keratocyst: a cyst, or a cystic neoplasm? J Dent Res 2011; 90: 133-42.

[3] Chi AC, Owings JR, Muller S. Peripheral odontogenic keratocyst: report of two cases and review of the literature. Oral Surg Oral Med Oral Pathol Oral Radiol Endod 2005; 99: 71-8.

[4] Valter K, Pavelic B, Katanec D, et al. Evaluation of sporadic cases of odontogenic keratocysts in multicentric study. Coll Antropol 2002; 26 Suppl: 177-82.

[5] Tanaka K, Yunoki H, Muroi Y, Haniwa H, Horii K, Kakudo K. A case of a lesion of the cheek histopathologically resembling keratocystic odontogenic tumor. Jpn J Oral Maxillofac Surg 2008; 43: 440-4.

[6] Precheur HV, Krolls SO. An unusual presentation of an odontogenic keratocyst in the buccal space: case report. J Oral Maxillofac Surg 2009; 67: 2513-25.

[7] Ide F, Kikuchi K, Miyazaki Y, Mishima K, Saito I, Kusama K. Keratocyst of the buccal mucosa: is it odontogenic? Oral Surg Oral Med Oral Pathol Oral Radiol Endod 2010; 110: e42-7.

[8] Ide F, Horie N, Shimoyama T, Saito I, Tanaka A, Kusama K. Infrequent clinicopathologic features of keratocystic odontogenic tumour: a 29-year multi-institutional retrospective review. Oral Surg 2008; 2: 1-9.

[9] Koizumi Y. Odontogenic keratocyst, orthokeratinized odontogenic cyst and epidermoid cyst: an immunohistochemical study including markers of proliferation, cytokeratin and apoptosis related factors. Int J Oral Med Sci 2004; 2: 14-22.

[10] Barr RJ, Headley JL, Jensen JL, Howell JB. Cutaneous keratocysts of nevoid basal cell carcinoma syndrome. J Am Acad Dermatol 1986; 14: 572-6.

[11] Baselga E, Dzwierzynski WW, Neuburg M, Troy JL, Esterly NB. Cutaneous keratocyst in naevoid basal cell carcinoma syndrome. $\mathrm{Br}$ J Dermatol 1996; 135: 810-2.

[12] Cassarino DS, Linden KG, Barr RJ. Cutaneous keratocyst arising independently of the nevoid basal cell carcinoma syndrome. Am J Dermatopathol 2005; 27: 177-8.

[13] Fernandez-Flores A. Cutaneous keratocyst: a renaming as isthmicanagenic cyst proposal. Am J Dermatopathol 2008; 30: 87-9.

[14] Kurokawa I, Nishimura K, Hakamada A, et al. Cutaneous dermoid cyst: cytokeratin and filaggrin expression suggesting differentiation towards follicular infundibulum and mature sebaceous gland. Oncol Rep 2006; 16: 295-9.

[15] Hovorakova M, Lesot H, Peterka M, Peterkova R. The developmental relationship between the deciduous dentition and the oral vestibule in human embryos. Anat Embryol 2005; 209: 303-13.

[16] Hovorakova M, Lesot H, Vonesch JL, Peterka M, Peterkova R. Early development of the lower deciduous dentition and oral vestibule in human embryos. Eur J Oral Sci 2007; 115: 280-7.

[17] Isomura ET, Okura M, Ishimoto S, et al. Case report of extragingival peripheral ameloblastoma in buccal mucosa. Oral Surg Oral Med Oral Pathol Oral Radiol Endod 2009; 108: 577-9.

[18] El Sedfy Bakry N. An ectopic odontome in the cheek. Oral Surg Oral Med Oral Pathol 1977; 43: 583-4.

(c) Yamamoto et al.; Licensee Bentham Open.

This is an open access article licensed under the terms of the Creative Commons Attribution Non-Commercial License (http://creativecommons.org/licenses/by-nc/3.0/) which permits unrestricted, non-commercial use, distribution and reproduction in any medium, provided the work is properly cited. 